\title{
SURFACES IN 3-SPACE THAT DO NOT LIFT TO EMBEDDINGS IN 4-SPACE
}

\author{
J. SCOTT CARTER \\ University of South Alabama, Mobile, Alabama 36688, U.S.A. \\ E-mail: carter@mathstat.usouthal.edu \\ MASAHICO SAITO \\ University of South Florida, Tampa, Florida 33620, U.S.A. \\ E-mail: saito@math.usf.edu
}

\begin{abstract}
A necessary and sufficient condition for an immersed surface in 3 -space to be lifted to an embedding in 4-space is given in terms of colorings of the preimage of the double point set.

Giller's example and two new examples of non-liftable generic surfaces in 3-space are presented. One of these examples has branch points. The other is based on a construction similar to the construction of Giller's example in which the orientation double cover of a surface with odd Euler characteristic is immersed in general position. A similar example is shown to be liftable with an explicit lifting given.

The problem of lifting is discussed in relation to the theory of surface braids. Finally, the orientations of the double point sets are studied in relation to the lifting problem.
\end{abstract}

1. Introduction. Consider a closed surface $M$ that is generically immersed in 3dimensional Euclidean space. Each point $x \in M$ has a neighborhood $N(x)$ such that the derivative of the immersion $f: M \rightarrow \mathbf{R}^{3}$, when restricted to $N(x)$ is injective; thus $\left.D f\right|_{N(x)}$ is of maximal rank and $f$ is one-to-one on $N(x)$. On the other hand, such a map may have multiple points that form the set $\left\{y \in \mathbf{R}^{3}: \# f^{-1}(y)>1\right\}$ which is the image of an immersed 1-dimensional manifold.

The multiple point set is stratified into two sets: the double point set is the set $S_{2}(f)=\left\{y \in \mathbf{R}^{3}: \# f^{-1}(y)=2\right\}$; the triple point set is the set $S_{3}(f)=\left\{y \in \mathbf{R}^{3}\right.$ : $\left.\# f^{-1}(y)=3\right\}$.

The immersion is called generic because the image of $f$ intersected with a neighborhood of a point in $S_{j}(f)$ looks like the intersection of $j$ coordinate planes in $\mathbf{R}^{3}$, and any

1991 Mathematics Subject Classification: 57Q45.

Key words and phrases: knotted surfaces, orientations, Boy's surface, triple points.

The paper is in final form and no version of it will be published elsewhere. 
smooth immersion may be perturbed into a generic immersion.

An immersed surface $f: M \rightarrow \mathbf{R}^{3}$ lifts to an embedding in $\mathbf{R}^{4}$ if there is an embedding $F: M \rightarrow \mathbf{R}^{4}$ and a projection $p: \mathbf{R}^{4} \rightarrow \mathbf{R}^{3}$ such that $p \circ F=f$. A well known consequence of Whitney's Theorem on normal Euler classes is the following [11]:

1.1. Theorem. If the Euler characteristic, $\chi(M)$ of the surface $M$ is odd, then for any immersion $f: M \rightarrow \mathbf{R}^{3}$ there is no lift $F: M \rightarrow \mathbf{R}^{4}$ of $f$.

Proof. If $F: M \rightarrow \mathbf{R}^{4}$ is an embedding, then the normal Euler class, $e(F)$ satisfies the congruence $e(F) / 2 \equiv \chi(M) \quad(\bmod 2)$. In particular, if $\chi(M)$ is odd, then the normal Euler class is non-trivial. This means that the normal bundle of $F$ does not have a section. But if $f(M)$ is a projection of $F(M)$, then the projection direction provides a section of the normal bundle of $F$.

An alternative proof of non-liftability of odd Euler characteristic surfaces for generic immersions will be given in Section 3. This proof is combinatorial and uses the Banchoff formula $\chi(F) \equiv T(f)(\bmod 2)$ where $T(f)=\# S_{3}(f)$ - the number of triple points of $f$ (cf. Francis [9]). In fact, the relationships among characteristic classes, multiple points, and singularities are rich and provide good geometric understanding of algebraic topological invariants.

It is natural to ask if there are orientable surfaces immersed in $\mathbf{R}^{3}$ that do not lift to embeddings. An example was provided by Giller [11]: The orientation double cover of Boy's immersion of the projective plane, when pushed into general position, does not lift to an embedding in 4-space. The idea in Giller's example is that the double arcs that return to certain triple points several times cannot maintain a global notion of over and under along them. Giller's proof is cumbersome since it depends on examining all possible choices of lifts at each of the eight triple points.

Giller's example had been puzzling us for many years. Why does the double cover of Boy's surface happen to be unliftable? Is it always the case that the double cover of non-orientable surfaces of odd Euler characteristic unliftable? Does a generic surface with branch points always lift if it has no closed loops in its double point set?

In this paper, we give answers to these questions, as well as new constructions of unliftable surfaces. The paper is organized as follows. In Section 2.3 we review Giller's construction, and give an example of an immersed non-orientable surface of genus 3 whose double cover lifts. Thus the answer to the second question above is negative. In Section 3, we give a necessary and sufficient criterion for immersed surfaces to be lifted. The condition is given in terms of colorings of the pre-images of the double point set, significantly simplifying Giller's proof. This answers the first question above in a combinatorial way. Using this condition we present a new example of an unliftable immersion constructed from a higher genus non-orientable surface.

In Section 4 we discuss orientations of the double point sets in relation to the lifting problem. Combining this and the methods used in the braid theory in dimension 4 , we give a generic surface with branch points that also does not lift in Section 5. Thus the answer to the third question is negative. 
2. Giller's construction and some examples. In this section we review some examples of immersed surfaces. We start with a fundamental definition.

2.1. Definition. Let $f: M \rightarrow \mathbf{R}^{3}$ denote an immersion of a closed surface into 3 -space. The preimage of the double point set,

$$
\{x \in M: f(x) \in S(f)\},
$$

is called the double decker set. The double decker set consists of generically immersed closed curves in $M$ on which there is a fixed point free involution. The double points in the double decker set are the pre-images of the triple points of $f$. These pre-images form the triple decker set. Thus the double decker set is the image of circles having double crossing points in the image. The image of each connected component of these circles is called a transverse component (or simply component for short).

2.2. Boy's surface. An illustration of Boy's surface (with a disk cut away so that singularities can be easily seen) is given in Figure 1. This is a classical immersion of a projective plane in 3-space. Apery's book [1] contains a plethora of alternative illustrations of this surface. The double decker set as it sits in a Möbius band is illustrated in Figure 2.

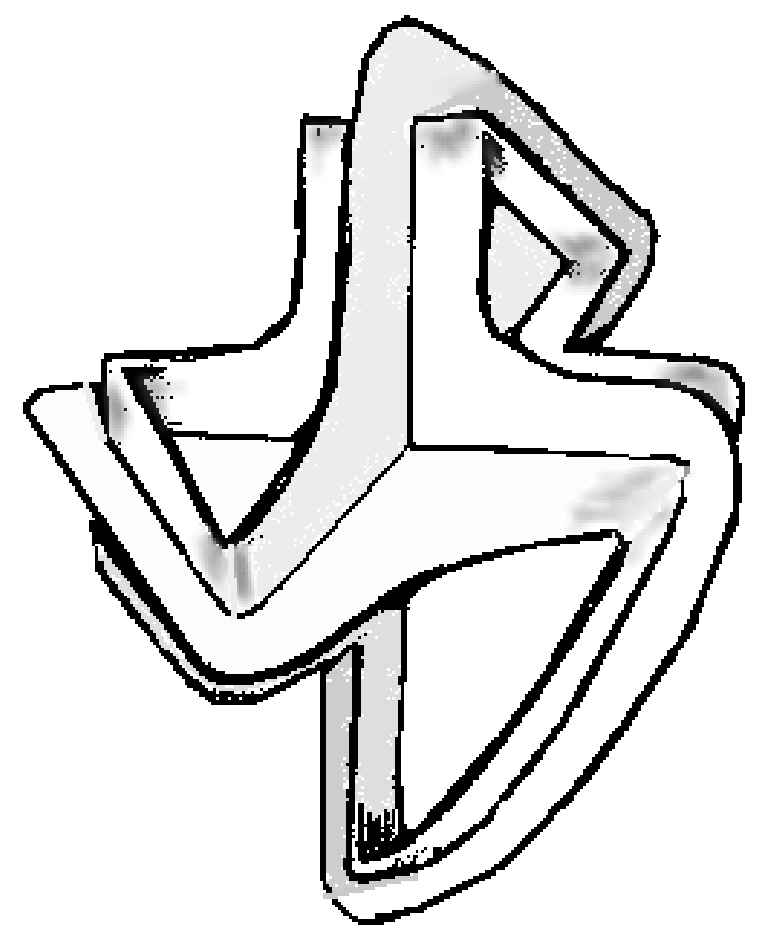

Fig. 1: Boy's surface

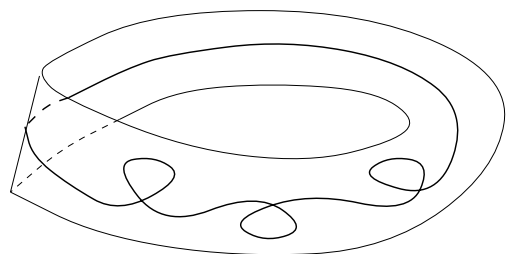

Fig. 2. The double decker set of Boy's surface

2.3. Giller's example. In Figure 3, an illustration of an immersed sphere is given. This is the result of perturbing the double cover of Boy's surface into general position. 


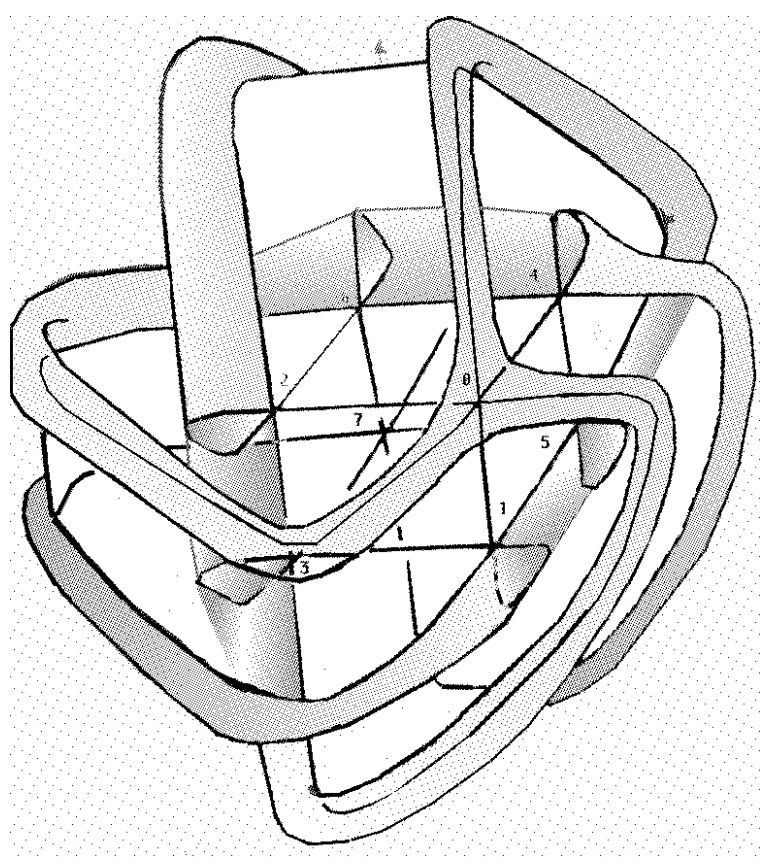

Fig. 3: The double cover of Boy's surface: Giller's example

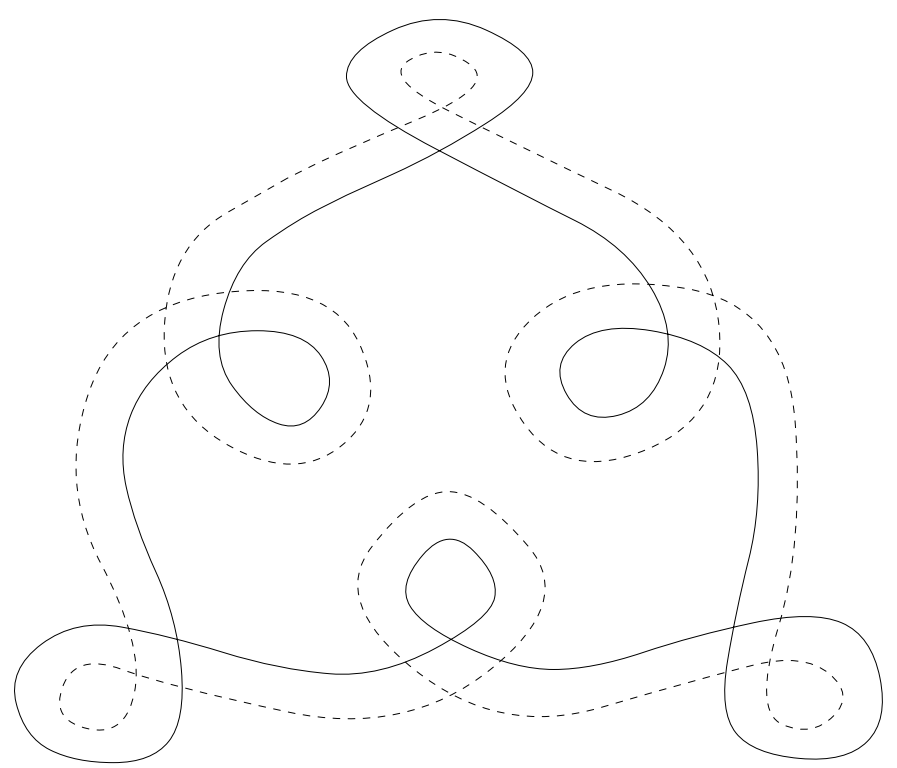

Fig. 4: The double decker set of Giller's surface

(To facilitate visualization, we have turned one of the disks that cover the missing disk in Figure 1 inside-out.) The resulting surface has eight triple points and a single double point curve that wraps through the triple points. This immersed sphere is Giller's Example. 
The double decker set for Giller's example is depicted in Figure 4. We have colored one component of the double decker set by using a dotted arc. A method for obtaining this double decker set is obtained as follows. Consider the double cover of the Möbius band embedded in 3-space by an annulus. Figure 2.7 of [3] contains an illustration. To construct a physical model of this surface, take the usual paper strip model of a Möbius band, but replace the single strip of paper with two strips. After the half twist is inserted, the two strips are taped end to end to each other. If the double decker set of Boy's surface is indicated on the Möbius band, then the double decker set of the un-perturbed double cover is that closed curve that maps to the double decker set of Boy's surface under the covering projection. The double decker set for Giller's example is obtained from this curve by pushing off a copy of it in the normal direction of the curve on the surface.

It is interesting to note that the two components (as manifolds) of the double decker set of Giller's example are interchangeable. That is, there is a homeomorphism of the 2 -sphere that takes one component to the other. Giller's example is sometimes used as an intermediate step to a sphere eversion which exploits this symmetry and the existence of Boy's surface.

2.4. Koschorke's example. In [16] Koschorke constructed an immersion of the connected sum of 3-copies of the projective plane with one triple point. The double point manifold has two components; one is immersed as a figure 8, and the other has a quarter twist along the framing induced by the immersion. The construction is given by taking a figure 8 times an interval, putting in a quarter twist, and capping off the boundary with a disk that intersects the $8 \times[0,1]$ in a figure 8 . Figure 5 illustrates this. The double decker set as it sits on a rank 3 non-orientable surface with one boundary component is illustrated in Figure 6.

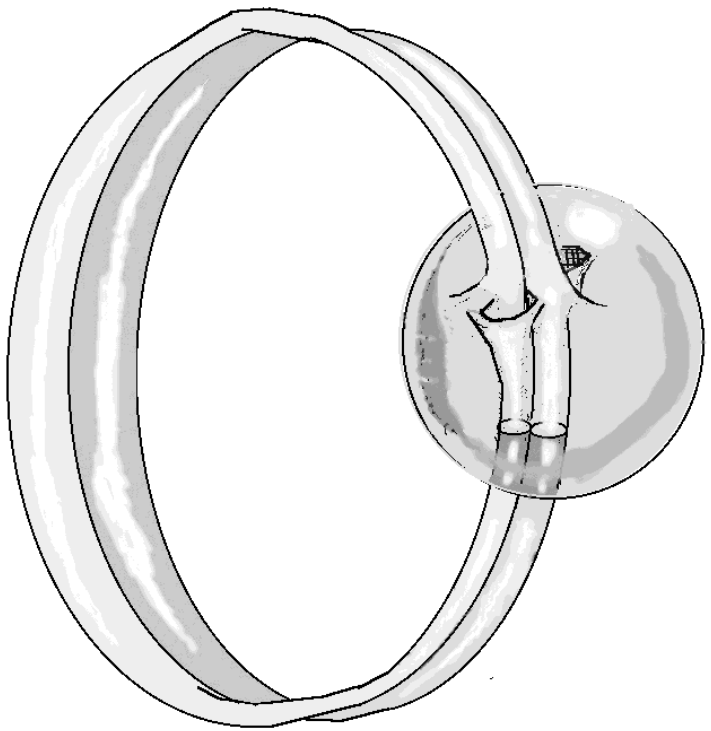

Fig. 5: Koschorke's surface

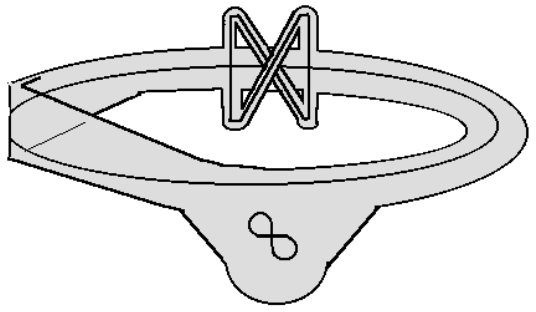

Fig. 6. The double decker set of Koschorke's surface 
To contrast with Giller's example, we show how to lift the double cover of Koschorke's example into 4-space. In Figure 7 an illustration of the critical portion of the double cover of Koschorke's surface is illustrated as a broken surface diagram. The crossing information in this figure is adequate to find a lift of the double cover of Koschorke's surface into 4space. The double decker set as it is immersed in the double cover (a surface of genus 2) of the double cover is indicated in Figure 8. Theorem 3.2 shows that there is a lift, and the choice of above and below sheets can be easily made to coincide with this illustration.

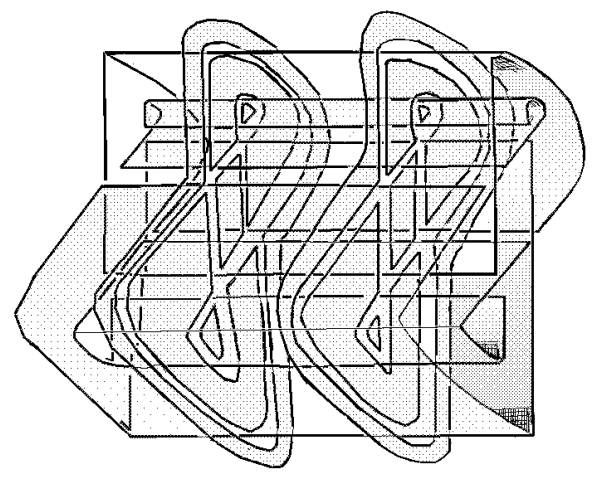

Fig. 7. The double cover of Koschorke's surface lifted

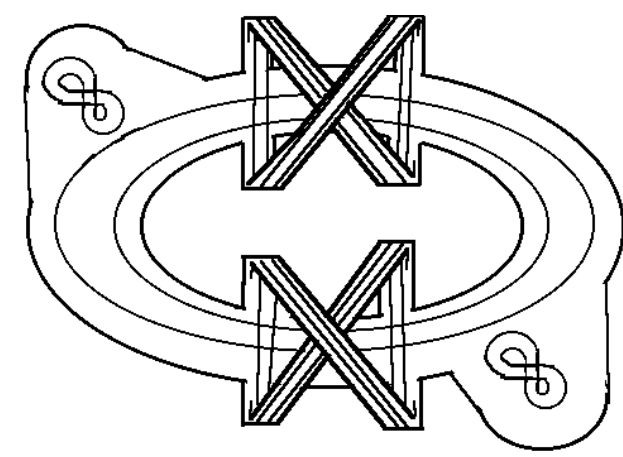

Fig. 8. The double decker set of the cover of Koschorke's surface

In the next section we will prove that Giller's example does not lift to an embedding.

3. Colorings of decker curves and liftability. We give a necessary and sufficient condition for immersed surfaces to be liftable.

Let $f: M \rightarrow \mathbf{R}^{3}$ be an immersion, $B$ be the double point curve, $D$ be the double decker curves.

3.1. Definition. The double decker curve $D$ is colorable if the components of $D$ can be divided into two families $\left\{D_{1}^{a}, \cdots D_{n}^{a}\right\}$ and $\left\{D_{1}^{b}, \cdots D_{n}^{b}\right\}$, (called $a$-curves and $b$-curves respectively), such that

(1) $D_{i}^{a}$ and $D_{i}^{b}$ are identified by $f$, and

(2) for every triple point $T$, the preimage of $T$ in $M$ consists of three crossing points between an $a$-curve and $a$-curve, an $a$-curve and $b$-curve, and a $b$-curve and $b$-curve.

These crossing points are called type $a / a, a / b$, and $b / b$ respectively.

3.2. Theorem. An immersion $f$ is liftable if and only if the decker set is colorable.

Proof. If $f$ is liftable, then the decker curves are colorable by declaring that the upper decker curves are $a$-curves and the lower curves are $b$-curves. Conversely, if $D$ is colorable, let $N$ be a thin tubular neighborhood of $b$-curves, and let $M^{\prime}=M \backslash \operatorname{Int}(N)$. Then $\left.f\right|_{M^{\prime}}$ gives a broken surface diagram representing a projection of an embedded surface. We give a review of the broken surface diagram in the following. 
First, recall that a classical knot diagram is obtained by removing the under path from the projection, and the knot can be recovered from the diagram by adding the under-arc in the lower half-space. In a broken surface diagram of a knotted surface, the portion of the surface that is further from the hyperplane of projection is broken. (Figure 7 contains a portion of a broken surface diagram and Figure 13 contains a broken surface diagram near a triple point.) We remove a small open tubular neighborhood of the $b$-curve and embed the resulting surface with boundary into 3-space using the restriction of $p \circ K$, where $K: M \rightarrow \mathbf{R}^{4}$ is an embedding. Conversely, for a given broken surface, we can reconstruct an embedding in 4-space that is isotopic to the given knot $K(F)$, by gluing the neighborhood of the lower-deck to the embedded surface in 3-space: The boundary of this neighborhood lies in 3-space, and the interior protrudes "below" this hyperplane - in other words, the neighborhood lies in the same component of $\mathbf{R}^{4} \backslash \mathbf{R}^{3}$ that $K(F)$ does.

We remark here that the idea of coloring surfaces was also used in videos $[10,19]$. In these videos, actual colors were used to indicate over- and under-crossings.

3.3. Proposition [11]. The double cover of Boy's surface is unliftable.

Proof. In Figure 4 the double decker set of the immersion $f$ which is the double cover of the Boy's surface is illustrated. There are parallel immersed curves, $A$ and $B$ say, in $S^{2}$ such that there are 6 intersection points between $A$ and $A, 6$ between $B$ and $B, 12$ between $A$ and $B$, respectively.

Suppose it lifted. Then $A$ is colored by $a$ and $B$ is colored by $b$, say. Then there are 6 crossings of type $a / a, 6$ of type $b / b$, and 12 of type $a / b$, a contradiction.

This simple proof can be used to generalize this phenomenon to other immersed surfaces.

3.4. TheOREM. Suppose an immersion has a transverse component of the double decker curves which is homotopic to the center line of a Möbius band neighborhood and which has a self intersection (necessarily at a triple point). Then the double cover of it is unliftable.

Proof. (The illustrations in Figures 10 and 11 should help the reader follow the proof.) Let $K$ be the double point curve in the assumption. Let $H$ be a neighborhood of $K$ which is an immersed Möbius band. Then the double cover $f: M \rightarrow \mathbf{R}^{3}$ has a neighborhood $N$ which double covers $H$. Here $N$ is a subset of $M$ which is an immersed annulus in $M$. The decker curves in $N$ consists of immersed parallel curves $A$ and $B$ that are parallel to the core of the annulus, and segments $\{p\} \times I \subset S^{1} \times I$. Suppose $f$ lifted. Then one of the parallel curves must be colored by $a$, the other by $b$.

By assumption the annulus $N$ has a self intersection. Let $S$ be a square subset of $N$ where $N$ has the intersection. The double decker set looks like \# in this square. These arcs are parallel pairs of $a$ - and $b$-curves.

Let $p_{1}, p_{2}, p_{3}$, and $p_{4}$ be the crossing points of the double decker set in $S$. Two of these are of type $a / b$ and one for each of $a / a$ and $b / b$.

Consider the four triple points $T_{i}$ that are the image of these crossing points $p_{i}$. Let $p_{i}^{\prime}$ and $p_{i}^{\prime \prime}$ be the preimages of $T_{i}$ different from $p_{i}$. Then $p_{i}^{\prime}, p_{i}^{\prime \prime}$ are crossing points of $A$, 
$B$ and 4 segments $I_{j}^{\prime}, I_{j}^{\prime \prime}$. Two of these segments are colored by $a$ and the other two are colored by $b$. Among the 12 crossings 3 are of type $a / a, 3$ are of type $b / b$ and 6 are of type $a / b$, a contradiction.

3.5. ExAmple. The immersion that is depicted in Figure 9 is an immersion of a non-orientable surface with boundary. The bounding circle is contained on a standardly embedded genus- 2 handle body; any embedded disk bounded by this curve outside of the genus 2 surface will intersect the original immersion in a pair of figure 8 curves. This example indicates a non-orientable surface that has 3 triple points. Figure 10 illustrates

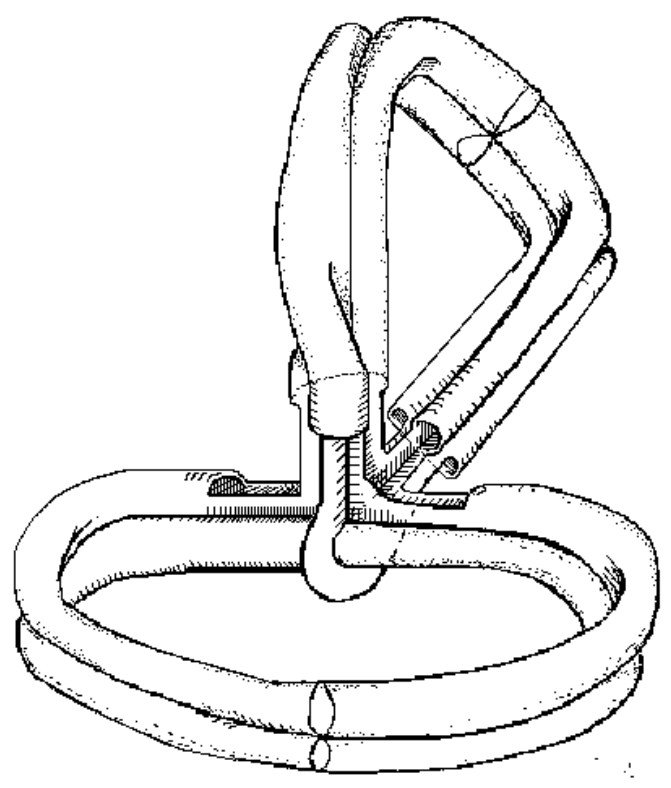

Fig. 9: A non-orientable surface with a figure 8 Möbius band

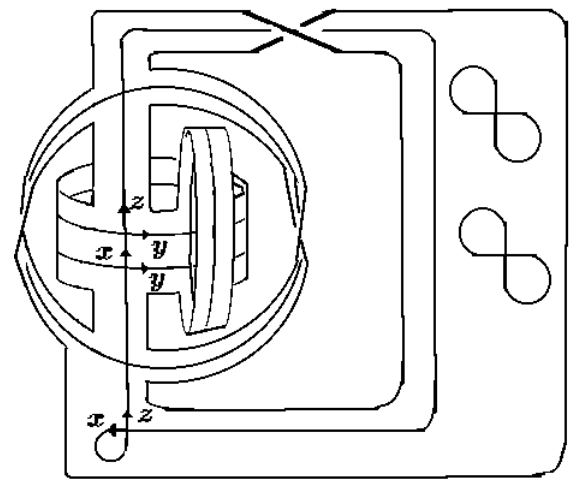

Fig. 10. The double decker set of the new surface

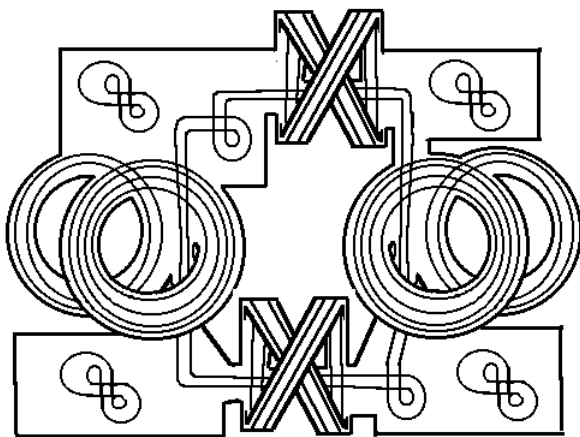

Fig. 11. The double decker set of the double cover of the new surface 
the double decker set as it sits on the ambient surface. This double decker set satisfies the hypothesis of Theorem 3.4. So the double cover of the example does not lift to 4-dimensions.

Figure 11 indicates the double decker set of the double cover, and indicates that the decker set is not colorable.

3.6. EXAMPLES. We observe that the coloring argument applies to any non-orientable curve of odd Euler characteristic. For if the Euler number is odd, then there must be an odd number of triple points by Banchoff's Theorem [2]: The number of triple points of an immersion is congruent to the Euler characteristic of the surface modulo 2. Banchoff's proof shows also that there must be an immersed Möbius band that contains an essential closed loop of double decker points if the Euler characteristic is odd. This component of the double decker manifold is a connected two-to-one cover of the corresponding double point curve, which does not satisfy the condition (1) of the colorability.

4. Orientations of double point sets. In this section, we indicate that for orientable surfaces there is an alternative criterion that is necessary and sufficient for liftability.

The criterion for liftability that we establish in this section will also apply to generic surfaces. A generic surface [4] has branch points in addition to double and triple points. It is called generic because any map from a surface into 3 -space can be perturbed into one with such image. A branch point has a neighborhood in which the surface intersects in a cone on a figure 8 (also known as a Whitney's umbrella). The middle-top picture in Figure 14 depicts a branch point.

Let $M$ denote an oriented surface and $f: M \rightarrow \mathbf{R}^{3}$ denote a generic map of $M$. Except at branch points there is an orientation normal vector assigned to each point at $f(M)$.

4.1. Definition. A distinguished region of $\mathbf{R}^{3} \backslash f(M)$ at a triple point is one of eight regions near the triple point into which all the normal vectors point. Distinguished edges of the double point curves near a triple point are the three (among six) edges sharing the triple point that are contained in the closure of the distinguished region.

4.2. Definition. An orientation of the double point set is an orientation of (transverse) components of it. An orientation of the double point set is compatible if the orientation restricted to edges at every triple point satisfy the following property: the number of the distinguished edges whose orientations point towards the triple point is exactly one, or exactly two.

A few explanations are in order. Note that there are six edges near each triple point. An orientation of them is given to transverse components, so that three of them go out and the other three go into the triple point. The compatibility condition says that the edges incident to the distinguished region can not be all going out nor all going in. Note also that the orientation can be naturally defined for generic surfaces. Oriented double point curves end at branch points in this case.

4.3. TheOREM. A generic surface lifts to an embedding in 4-space if and only if the double point curves admit a compatible orientation. 
Proof. Let $x$ be a double point in $f(M)$ and $\gamma$ be a part (homeomorphic to an interval) of the double point curve. Suppose $f$ lifts to an embedding. Then at each double point $x$, one of the sheets forming the double points is up above the other sheet with respect to the projection direction. (In terms of the coloring of the preceding Section, the sheet with $a$-curve is the upper sheet and one with $b$-curve is the lower sheet.)

Give an orientation to $\gamma$ so that (the orientation of $\gamma, v_{0}, v_{1}$ ) matches the orientation of the 3 -space by the right hand convention, where $v_{0}$ (resp. $v_{1}$ ) is the normal to the upper (resp. lower) sheet.

One checks that this orientation satisfies the compatibility condition.

Conversely, a broken surface diagram can be constructed given a compatible orientation. Specifically, a local orientation of a double curve together with the orientations of two sheets involved, determines which sheet is above the other sheet. Then one checks that such over/under relations are well defined at a triple point if the orientations are compatible. Figure 13 depicts one of the cases.

4.4. EXAmple. In Giller's example, there is only one transverse component of the double point curve. Therefore there are only two choices to orient this curve. For either choice, the triple points labeled 0 and 7 do not have a compatible orientation.

4.5. R e mark (Signs of triple points). The compatibility condition for triple points discussed above says that there are exactly one or exactly two incoming distinguished edges. Thus we have two types of such triple points. If it has exactly one (resp. two) incoming distinguished edge(s) then call it a triple point of type I (resp. II). We observe that the types of triple points are related to the orientations of the double decker set defined by Mikhalkin and Polyak [17].

Let $M$ denote an oriented surface and $f: M \rightarrow \mathbf{R}^{3}$ denote an immersion of $M$. Choose an orientation of the normal bundle of $f$ such that $T(M) \oplus \nu$ agrees with the right handed orientation of $\mathbf{R}^{3}$. Consider a double point $y \in S_{2}(f)$. Suppose that the points $x_{1}, x_{2} \in M$ are pre-images of $y$ : Thus $f\left(x_{1}\right)=f\left(x_{2}\right)=y$. The double decker arcs $\gamma_{1}$ and $\gamma_{2}$ in $M$ that pass through $x_{1}$ and $x_{2}$ are embeddings $\gamma_{j}:(-\epsilon, \epsilon) \rightarrow M$ with $\gamma_{j}(0)=x_{j}$ and $f\left(\gamma_{j}(-\epsilon, \epsilon)\right) \subset S_{2}(f)$. The normal direction to $\gamma_{j}$ in $M$ is chosen so that its projection to $\mathbf{R}^{3}$ agrees with the direction that is normal to $f(M)$ at $x_{j+1}$ (subscripts read modulo 2). We let $v\left(x_{j}\right) \in T M_{x_{j}}$ denote this normal vector. Figure 12 indicates this convention. The local parametrizations of the double decker curves can be chosen so that the orientation $\left[\gamma_{j}^{\prime}(0), v\left(x_{j}\right)\right]$ agrees with the given orientation of $M$ at $x_{j}$. This orientation was defined by Mikhalkin and Polyak [17].

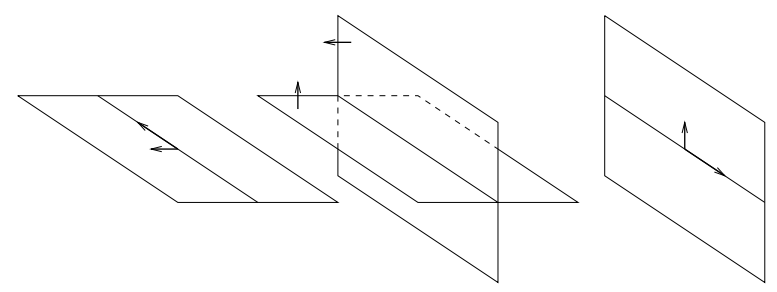

Fig. 12: Mikhalkin-Polyak orientation 
If $f(M)$ lifts, the double point curves admits the orientation defined in the proof of Theorem 4. The relation between this orientation and the above defined orientation is as follows. Two orientations agree for lower decker curves and disagree for upper decker curves.

For each preimage of three sheets at a triple point, define the signs as follows. First note that the decker curves have the order on each sheet. On the top (resp. middle, bottom) sheet, the decker curve which is the intersection with the middle (resp. top, top) sheet is upper and that with the bottom (resp. bottom, middle) sheet is lower. If the orientation in the sense of Mikhalkin-Polyak of (upper deck, lower deck) matches the orientation of the surface, then call it positive (otherwise negative). Thus we get a triple of signs $( \pm, \pm, \pm)$ for each of crossings of decker curves on the top, middle, and bottom sheets assigned to each triple point.

We use the following convention. Break the lower decker curve into two arcs. Then the decker curves look like a classical knot crossings. Then the sign of each sheet is the sign of the crossing viewed from the positive side of the surface. Figure 13 indicates this situation.

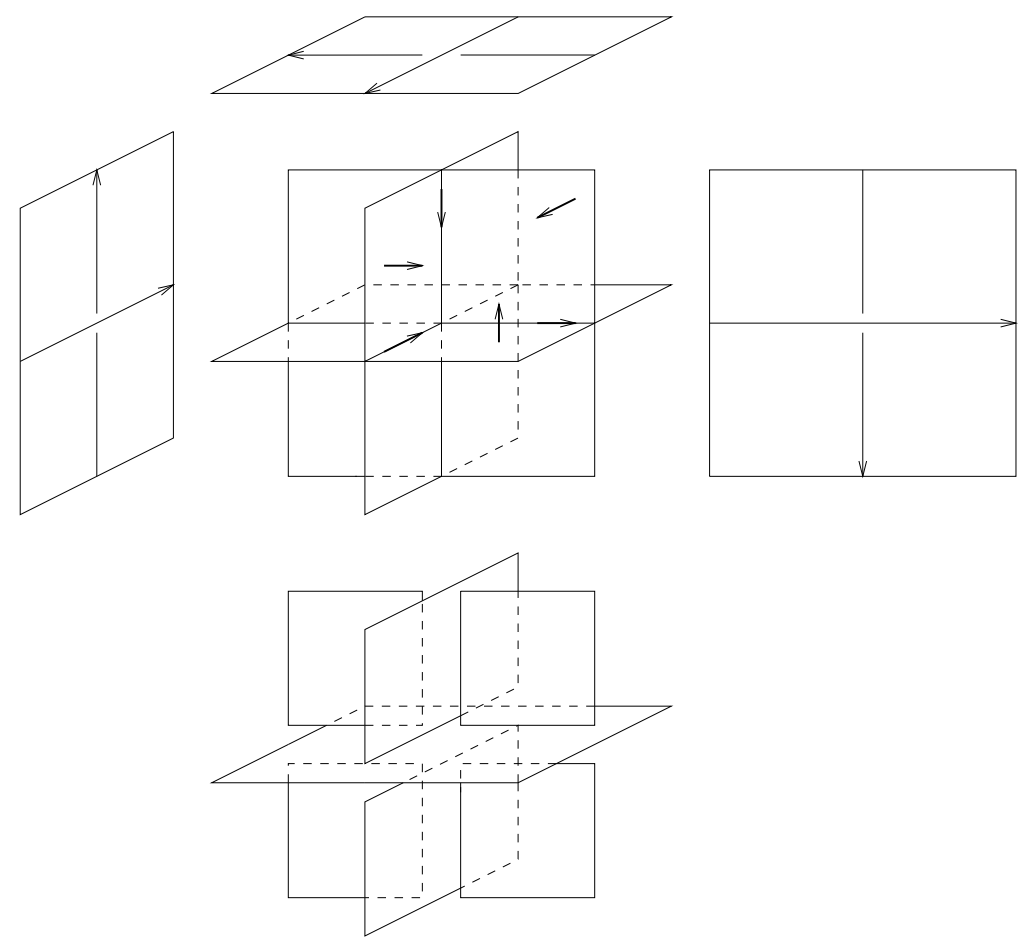

Fig. 13: Types of triple points and orientations

4.5.1. Proposition. The signs of any triple point is either $(+,-,+)$ or $(-,+,-)$.

Pr o of. Figure 13 depicts a neighborhood of a triple point. The normal vectors of each sheet is indicated by short arrows pointing out from each sheet. The broken surface 
diagram of this neighborhood is also depicted. Surrounding the picture of the triple point are pre-images of the three sheets forming the triple point and the decker curves. The lower decker curves are broken. The orientations of decker curves are those of MikhalkinPolyak. In this Figure, the top sheet (the horizontal sheet) has the sign - between decker curves. The middle (reps. bottom) sheet has sign + (resp. -). Thus in this case the signs are $(-,+,-)$. If one of the orientations, say that of the top sheet, of the surface is changed, then the orientations of decker curves on the middle sheet and the bottom sheet that are the intersections with the top sheet will change. Thus the new signs are $(+,-,+)$. All the cases are obtained by changing orientations and a change will cause the change of all the signs.

4.5.2. Proposition. A triple point is of type I (resp. II) iff it has signs $(+,-,+)$ (resp. $(-,+,-))$. Moreover, for a type I (resp. II) triple point the orientation (normal to top, normal to middle, normal to bottom) agrees (resp. disagrees) with the right handed orientation of 3-space.

Pr o of. In Figure 13, the orientations of the double point curves are also depicted. Thus in this case the triple point is of type II. If one of the orientations are changed the type changes, so do the signs as observed above. Thus the Proposition follows.

5. Surface braids and the lifting problem. In this section we give a new construction of unliftable generic surfaces.

Figure 14 shows how to express certain generic surfaces in 3-space by means of planar graphs and by sequences of words in symmetric groups. These descriptions in fact came from the theory of surface braids in 4-space, generalizations of the Artin's braid groups to 4 -dimensions. We refer interested readers to $[5,6,7,14]$ for example. Let us explain these descriptions.

We consider a surface $F$ in a box $B=I_{1} \times I_{2} \times I_{3} \subset \mathbf{R}^{3}$ a schematic of which is depicted on the right of Figure 14, where $I_{j}$ denotes a copy of the unit interval, for $j=1,2,3$. We require that the surface $F$ in $B$ satisfies the following conditions.

- $F$ is generic.

- The boundary $\partial F$ of $F$ is contained in $\partial\left(I_{1} \times I_{2}\right) \times I_{3}$.

- The projection $p: I_{1} \times I_{2} \times I_{3} \rightarrow I_{1} \times I_{2}$ restricted to $F$ is a branched covering such that each branch point is of degree 2 .

Let $D$ be the double point set of $F$. Then $p(D) \subset I_{1} \times I_{2}$ is a planar graph. Let us call this an un-oriented chart. An un-oriented chart has univalent vertices corresponding to branch points, 4-valent vertices that correspond to the crossings of the projections of double arcs, and 6-valent vertices corresponding to triple points of $F$. A generic intersection $F \cap I_{1} \times\{t\} \times I_{3}$, consists of intersecting strings in $I_{1} \times\{t\} \times I_{3}$.

Such strings in a disk represent words in the symmetric group $S_{n}$ where each crossing represents a transposition $s_{i}=(i, i+1)$ (cf. [8]). Thus from $t=0$ to $t=1$, we get a sequence of words $\left.S=\left(w_{1}, w_{2}, \cdots, w_{k}\right)\right)$ in $S_{n}$ where $w_{1}$ and $w_{k}$ are empty words. Furthermore, one can take slices so that each change between $w_{j}$ and $w_{j+1}$ is of one of the following types. 

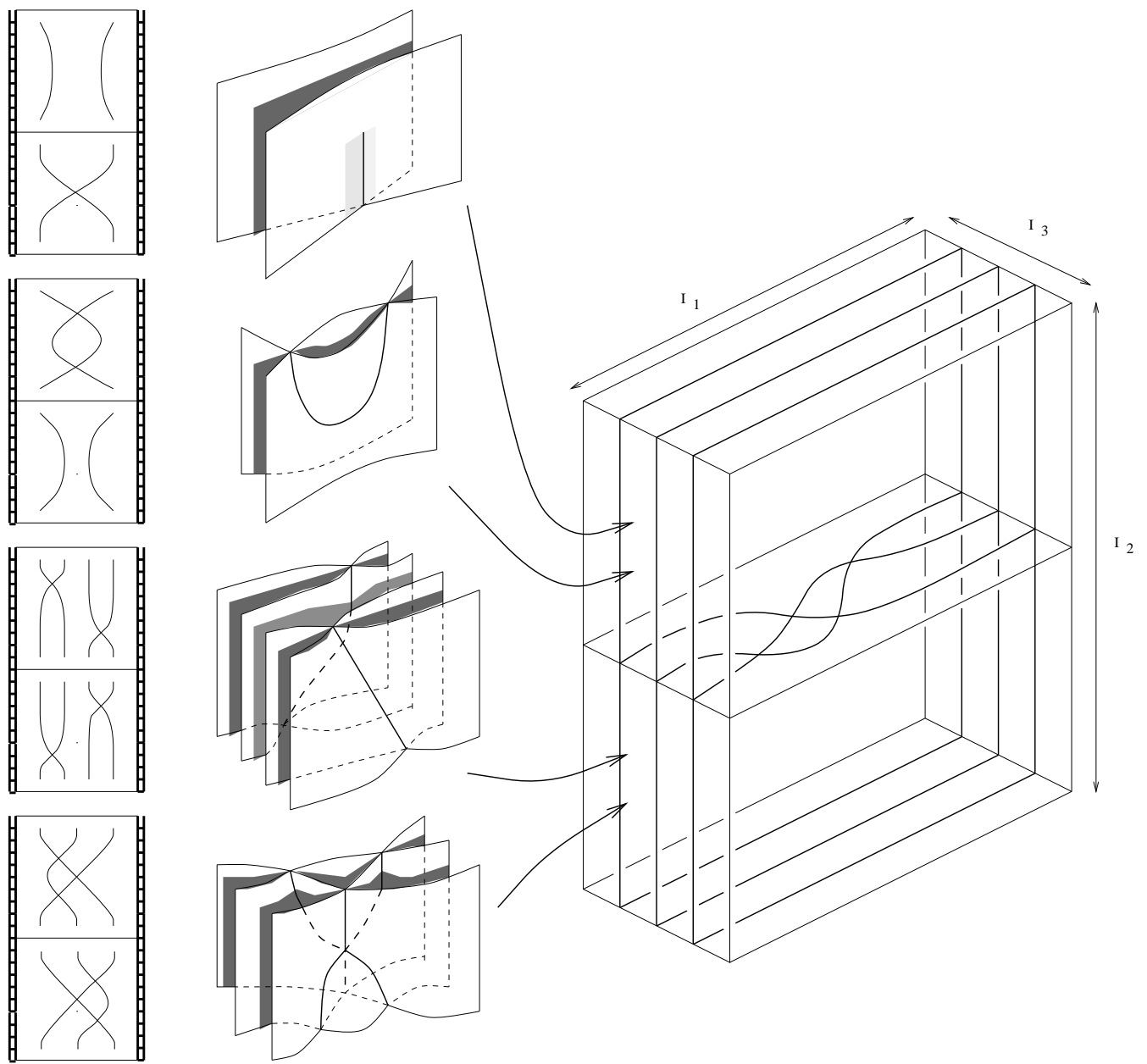

(1) Insertion/deletion of $s_{i}$,

(2) Insertion/deletion of $s_{i}^{2}$,

(3) Replacement of $s_{i} s_{j}$ by $s_{j} s_{i}$ where $|i-j|>1$,

(4) Replacement of $s_{i} s_{j} s_{i}$ by $s_{j} s_{i} s_{j}$ where $|i-j|=1$.

One can label each edge of the un-oriented chart by integers 1 through $n-1$ using the convention that each edge corresponds to a generator of symmetric groups. The univalent vertices of the chart correspond to the insertion or deletion of a transposition (item (1) above). The 6 -valent vertices correspond to the replacement (4). The 4 -valent vertices correspond to applying the replacement (3). And maximal/minimal points on the edges correspond to the insertion or deletion of the square of a transposition (item (2)). In summary, such surfaces in a box can either be represented by labeled un-oriented charts or sequences of words in the symmetric groups. 
If $F$ is a projection of a surface embedded in 4-space then the double point set has a compatible orientation as defined in Section 4. We can use the compatible orientation to obtain (oriented) charts as defined by Kamada [12, 14, 13].

Furthermore, the broken surface diagrams of $F$ in this case give rise to sequences of words in the braid group $B_{n}$ of $n$-strings. Then the changes between words are described by:

(1) Insertion/deletion of $\sigma_{i}^{\epsilon}$,

(2) Insertion/deletion of a pair $\sigma_{i}^{\epsilon} \sigma_{i}^{-\epsilon}$,

(3) Replacement of $\sigma_{i}^{\epsilon_{1}} \sigma_{j}^{\epsilon_{2}}$ by $\sigma_{j}^{\epsilon_{2}} \sigma_{i}^{\epsilon_{1}}$ where $|i-j|>1$,

(4) Replacement of $\sigma_{i}^{\epsilon} \sigma_{j}^{\epsilon} \sigma_{i}^{\epsilon}$ by $\sigma_{j}^{\epsilon} \sigma_{i}^{\epsilon} \sigma_{j}^{\epsilon}$ where $|i-j|=1$, where $\epsilon, \epsilon_{1}, \epsilon_{2}= \pm 1$. See [5] for more details.

5.1. Definition. A sequence of words on the generators of the braid group and their inverses that satisfies the condition that successive words differ by at most one of the changes (1)-(4) is called a braid movie.

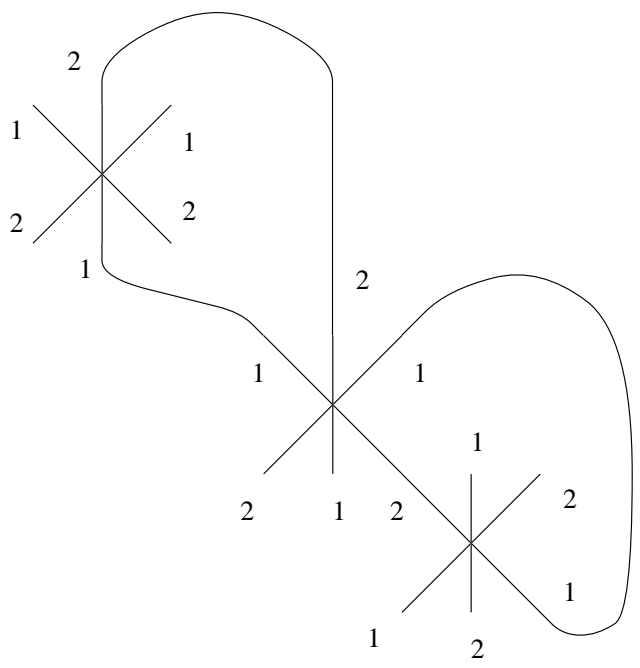

Fig. 15: A chart which does not lift

An example of an unoriented chart is given in Figure 15. This figure defines a generic orientable surface in 3 -space. We prove

5.2. Proposition. The generic surface F in 3-space described by Figure 15 does not lift to an embedded surface in 4-space.

Pr o of. Suppose $F$ lifts. Then there exists an orientation on every edge which satisfies the compatibility condition at every 6 -valent vertex (the image of a triple point). Let us define this condition.

Let $C$ be a small circle around a 6 -valent vertex. If we travel around $C$ in whichever direction, we have 6 intersection points between $C$ and the graph. Each edge has an orientation. We say that the orientation is compatible at the vertex if the orientations of 
the edges are in the order of (in, in, in, out, out, out) for a certain choice of starting point on $C$. Here in (resp. out) means that the orientation of the edge points inward (resp. outward) of $C$.

This compatibility condition follows from the compatibility condition defined in Section 4. Alternatively, one could check that if $F$ is a projection of an embedded surface then the induced orientation defined above satisfies the compatibility condition at every vertex by checking all the braid relations. The incompatible orientation does not realize braid relations without making crossing changes.

Thus it is necessary and sufficient for an un-oriented chart to represent a chart of embedded surface is to have an orientation which satisfies the compatibility condition at every vertex.

Notice that in the chart there is a transverse component of an arc, say $\Gamma$, which goes through the middle vertex three times. There are only two possible ways of giving orientations to each transverse components of arcs. In particular, $\Gamma$ has only two possible orientations. One easily checks that neither of them gives an orientation which satisfies the compatibility condition at the middle vertex.

In fact, by using the new compatibility condition we defined in the above proof, we have proved

5.3. THEOREM. The generic surface represented by an unoriented chart lifts to an embedding if and only if it admits a compatible orientation.

This example has the following consequence in terms of sequences of words in the symmetric group $S_{3}$ and the braid group $B_{3}$ with 3 -strings.

The chart in Figure 15 has the following sequence, $S$, of words in $S_{3}$ associated with it:

$\begin{array}{ll}\emptyset & \text { A square of } s_{2} \text { added } \\ s_{2}^{2} & \text { A generator } s_{1} \text { added } \\ s_{1} s_{2}^{2} & \text { A generator } s_{1} \text { added } \\ s_{1} s_{2} s_{1} s_{2} & \text { A relation performed } \\ s_{2} s_{1} s_{2} s_{2} & \text { The first letter deleted } \\ s_{1} s_{2} s_{2} & \text { The second letter deleted } \\ s_{1} s_{2} & \text { A square of } s_{1} \text { added } \\ s_{1} s_{2} s_{1}^{2} & \text { A relation performed } \\ s_{2} s_{1} s_{2} s_{1} & \text { The first letter deleted } \\ s_{1} s_{2} s_{1} & \text { The first letter deleted } \\ s_{2} s_{1} & \text { The second letter } s_{1} \text { added } \\ s_{2} s_{1} s_{1} & \text { The third letter } s_{2} \text { added } \\ s_{2} s_{1} s_{2} s_{1} & \text { A relation performed } \\ s_{1} s_{2} s_{1} s_{1} & \text { The first letter deleted } \\ s_{2} s_{1} s_{1} & \text { The first letter deleted } \\ s_{1} s_{1} & \text { The square of } s_{1} \text { deleted } \\ \emptyset & \end{array}$

5.4. Corollary. The above sequence $S$ does not lift to a braid movie in $B_{3}$. 
Recall that a braid movie is a sequence of words that satisfies the condition in Definition 5. A lift of such a sequence is a braid movie that projects to the given sequence under the map $\sigma_{i}, \sigma_{i}^{-1} \mapsto s_{i}$. The Corollary obviously follows from the Theorem.

5.5. An alternative proof of Theorem 5. The permutation movie (i.e., the sequence of symmetric group words given above) illustrated in Figure 16 indicates the cross sections of the generic surface. The double decker set for this surface is indicated in Figure 17.

First let us explain these figures. We capped off the properly embedded surface in $I_{1} \times I_{2} \times I_{3}$ with three nested disks to obtain a closed surface. Numbers are assigned to crossings in Figure 16. These numbers have no relation to the labelings of edges of the
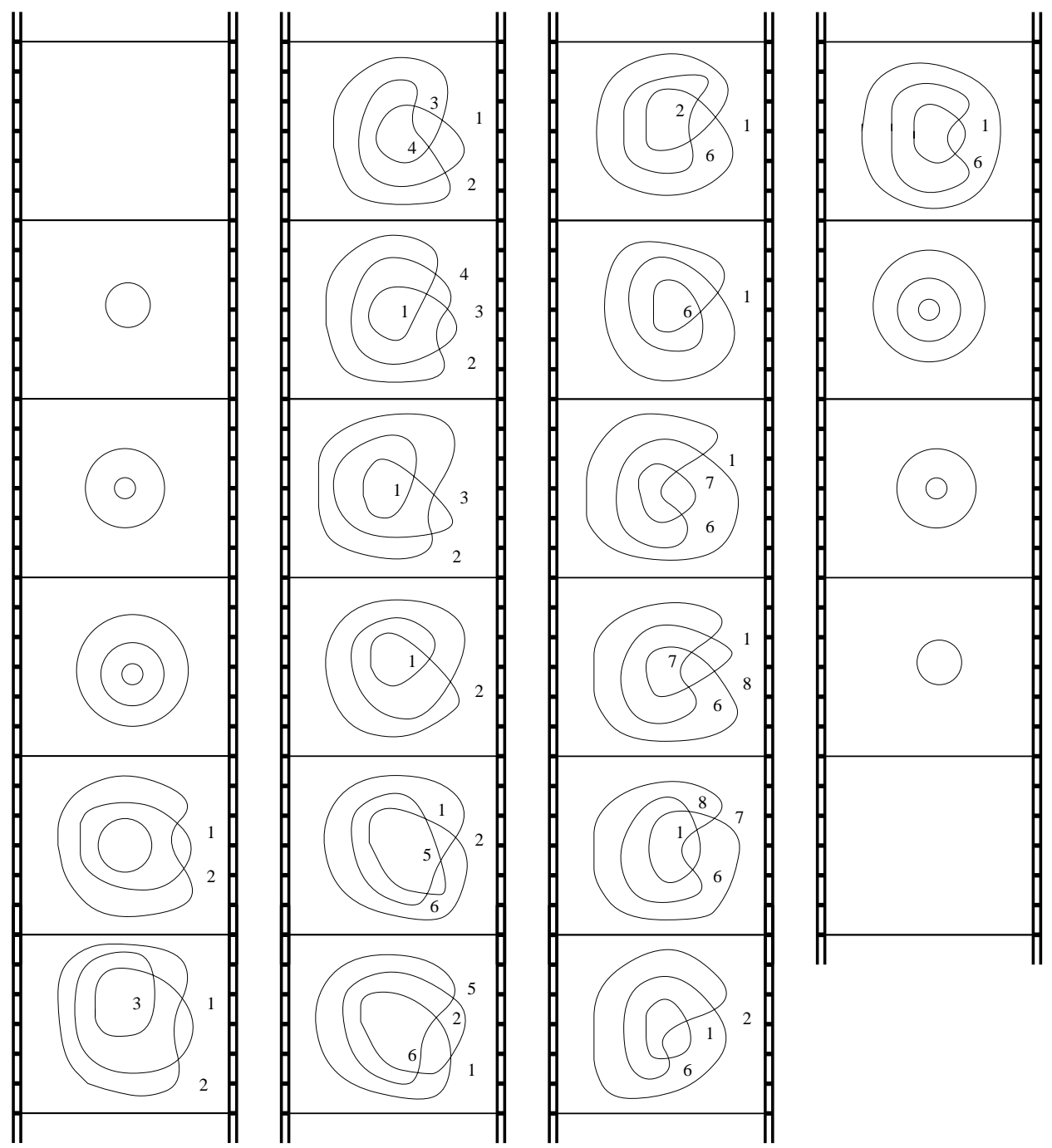

Fig. 16: A movie description of the unliftable surface 

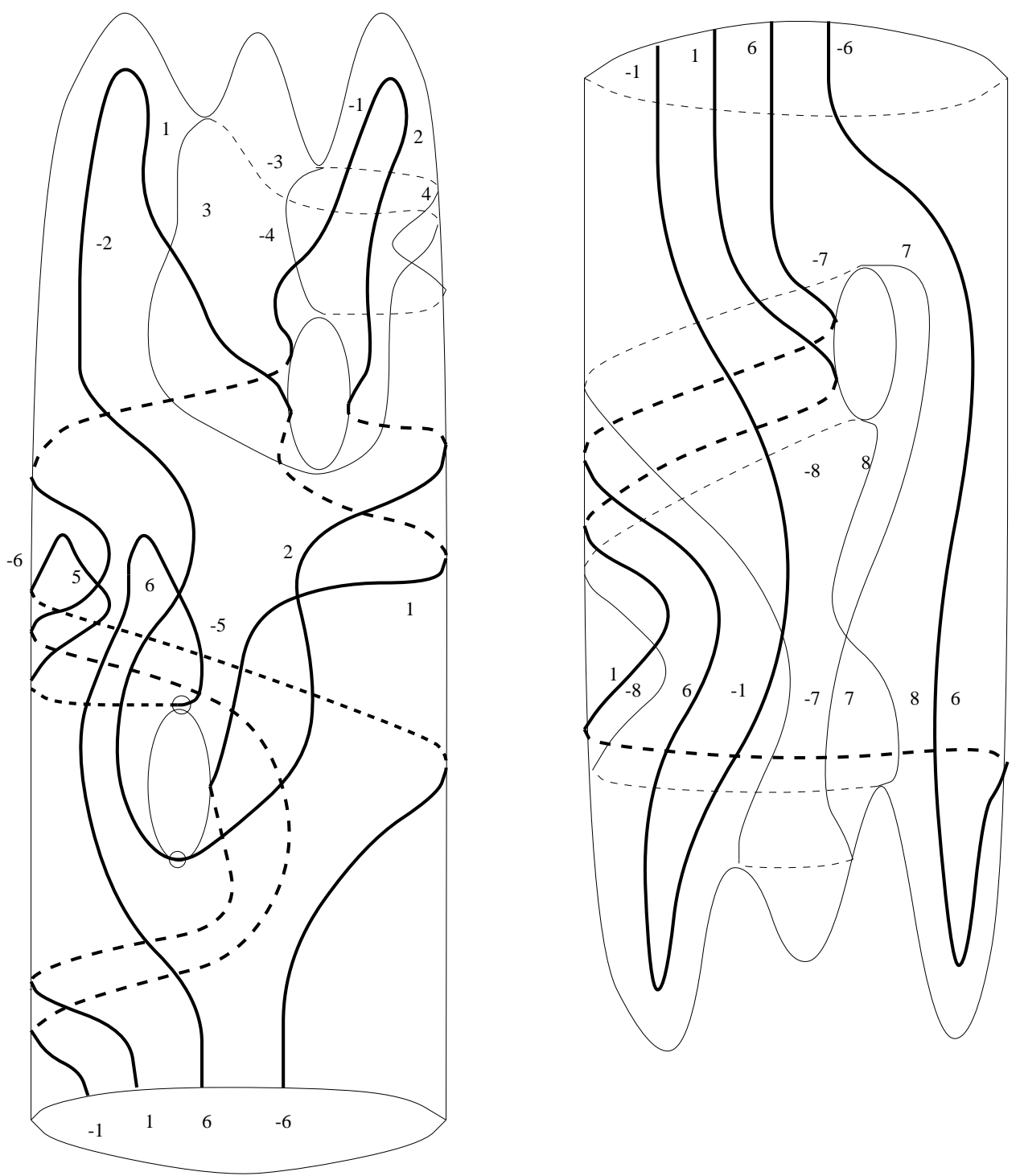

Fig. 17: The double decker curves of the unliftable surface

chart. The decker curves corresponding to the crossing point labeled $j$ are labeled by $\pm j$ in Figure 17. The long edge in the chart corresponds to the decker curve in Figure 17 in a thick line.

The decker curve was constructed as follows. First we have three circles in a still of the movie description that are represented by three maximal points in the left of Figure 17. The introduction of $s_{2}^{2}$ in a symmetric group word sequence are seen in the decker set as a pair of maximal points of decker curves labeled \pm 1 and \pm 2 . When a generator is introduced, two components of curves are connected; when a generator is deleted, two curves are connected. Thus at such a point we see a saddle point of the surface 
together with the decker curve passing through the saddle point. These correspond to (a) univalent vertices in the unoriented chart, (b) insertions/deletions of transpositions in the symmetric group movie. Two of these are indicated in the figure with small circles at the top and bottom of the second hole from the top.

Now the coloring condition can be generalized to generic surfaces as follows: the double decker curve $D$ is colorable if the components of $D \backslash$ (branch points) can be divided into two families $\left\{D_{1}^{a}, \cdots D_{n}^{a}\right\}$ and $\left\{D_{1}^{b}, \cdots D_{n}^{b}\right\}$, (called $a$-curves and $b$-curves respectively), such that

(1) $D_{i}^{a}$ and $D_{i}^{b}$ are identified by $f$, and

(2) for every triple point $T$, the preimage of $T$ in $M$ consists of three crossing points between an $a$-curve and $a$-curve, an $a$-curve and $b$-curve, and a $b$-curve and $b$-curve.

(3) two edges whose closure shares a branch point receive the opposite color (i.e., one is $a$ and the other is $b$ ).

One easily sees that this colorability is a necessary and sufficient condition for a generic surfaces to be lifted.

Going back to the example, there is a single loop represented by a thick line in the decker set. This has two branch points dividing the loop into two arcs. One must be colored $a$ and the other by $b$ by the condition (3), but the condition (2) is not satisfied by either of two possible colorings.

\section{References}

[1] F. A pery, "Models of the Real Projective Plane," Vieweg (Braunschweig 1987).

[2] T. F. Banchoff, Triple Points and Surgery of Immersed Surfaces, Proc. AMS 46, No.3 (Dec. 1974), 403-413.

[3] J. S. Carter, "How Surfaces Intersect in Space: an Introduction to Topology," World Scientific Publishing, 2nd edition (Singapore 1995).

[4] J. S. Carter and M Saito, Reidemeister Moves for Surface Isotopies and Their Interpretation as Moves to Movies, J. of Knot Theory and its Ram., vol. 2, no. 3, (1993), 251-284.

[5] J. S. Carter and M. Saito, Braids and Movies, J. of Knot Theory and its Ram. 5, no. 5 (1996), 589-608.

[6] J. S. Carter and M. Saito, Knotted Surfaces, Braid Movies and Beyond, in Baez, J., "Knots and Quantum Gravity," Oxford Science Publishing (Oxford 1994), 191-229.

[7] J. S. Carter and M. Saito, Knot Diagrams and Braid Theories in Dimension 4, Real and Complex Singularities, W. L. Marar (ed.), Pitman Res. Notes Math. Ser. 333, Longman Sci. Tech., Harlow, 1995, 112-147.

[8] J. S. Carter, D. E. Flath and M. Saito, "The Classical and Quantum 6j-symbols," Princeton University Press Lecture Notes in Math Series (1995).

[9] G. F. Francis, "A Topological Picturebook," Springer-Verlag (New York 1987).

[10] A. Hansen, Knot ${ }^{4}$, a video presented at SIGGRAPH '93. His software Meshview was used to produce this video.

[11] C. Giller, Towards a Classical Knot Theory for Surfaces in $\mathbf{R}^{4}$, Illinois Journal of Mathematics 26, No. 4, (Winter 1982), 591-631. 
[12] S. Kamada, Surfaces in $R^{4}$ of braid index three are ribbon, Journal of Knot Theory and its Ramifications 1 (1992), 137-160.

[13] S. Kamada, 2-dimensional braids and chart descriptions, "Topics in Knot Theory", Proceedings of the NATO Advanced Study Institute on Topics in Knot Theory, Turkey, (1992), 277-287.

[14] S. Kamada, A characterization of groups of closed orientable surfaces in 4-space, Topology 33 (1994), 113-122.

[15] S. Kamada, Generalized Alexander's and Markov's theorems in dimension four, Preprint.

[16] U. Koschorke, Multiple points of Immersions and the Kahn-Priddy Theorem, Math Z. 169 (1979), 223-236.

[17] G. Mikhalkin and M. Polyak, Whitney formula in higher dimensions, J. Differential Geom. 44 (1996), 583-594.

[18] D. Roseman, Reidemeister-type moves for surfaces in four dimensional space, this volume.

[19] D. Roseman, Twisting and Turning in Four Dimensions, A video made at the Geometry Center (1993). 